\section{Regards sur l'économie allemande}

Bulletin économique du CIRAC

$74 \mid 2005$

Varia

\title{
Industrie et ménages face au double choc : euro et coûts énergétiques
}

\section{Markus Gabel}

\section{(2) OpenEdition}

\section{Journals}

Édition électronique

URL : http://journals.openedition.org/rea/159

DOI : $10.4000 /$ rea. 159

ISBN : 978-2-8218-0844-7

ISSN : 1965-0787

\section{Éditeur}

CIRAC

Édition imprimée

Date de publication : 1 décembre 2005

Pagination : 9-16

ISSN : 1156-8992

\section{Référence électronique}

Markus Gabel, « Industrie et ménages face au double choc : euro et coûts énergétiques », Regards sur l'économie allemande [En ligne], 74 | décembre 2005, document 1, mis en ligne le 25 juin 2008, consulté le 20 avril 2019. URL : http://journals.openedition.org/rea/159; DOI : 10.4000/rea.159 


\title{
Industrie et ménages face au double choc : euro et coûts énergétiques
}

\author{
Markus Gabel
}

Depuis 2002, l'économie allemande est confrontée à deux chocs exogènes: d'abord l'appréciation de l'euro, ensuite la très forte augmentation des cours du pétrole, amenant tous les prix énergétiques dans une spirale ascendante. Mais les entreprises résistent étonnamment bien: l'Allemagne a de fortes chances de décrocher pour la troisième année consécutive le titre de championne mondiale des exportations, permettant ainsi aux entreprises d'atteindre des bénéfices records malgré l'atonie de la demande intérieure. Cette extraordinaire résistance s'explique par le concours de plusieurs facteurs. C'est d'abord la Chine qui absorbe de plus en plus de biens allemands et contribue, comme les PECO, à contenir outre-Rhin les coûts de production grâce à ses livraisons de biens bon marché. Ensuite, les pays producteurs de pétrole réinjectent plus rapidement qu'autrefois la rente pétrolière et commandent beaucoup de produits d'investissement outre-Rhin. Mais plus fondamental est le fait que les entreprises allemandes ont nettement gagné en compétitivité. Une des raisons en réside dans les caractéristiques de l'Union économique et monétaire dont l'Allemagne profite actuellement plus que les autres membres de la zone euro: si les taux d'intérêt sont identiques dans l'ensemble de la zone euro, le taux d'inflation allemand, traditionnellement inférieur à celui des autres Etats membres, ralentit comparativement la progression du coût des produits allemands à l'export. Reste tout de même un sérieux risque pour la compétitivité : l'Allemagne s'offre le luxe d'avoir des prix d'électricité et de gaz parmi les plus élevés au monde.

Entre 2002 et 2004, l'appréciation de l'euro a beaucoup préoccupé les esprits. La monnaie européenne s'est appréciée de plus de $50 \%$ entre son niveau le plus bas à la fin 2000 et son plus haut à la fin 2004. Cette appréciation a pu être digérée par les entreprises allemandes grâce à la relative lenteur de son évolution (voir REA 63/03). Depuis, l'euro est revenu à des niveaux plus modérés ( 1,2 au lieu de 1,35 pour un dollar) et se situe maintenant près de son niveau médian sur 10 ans. Mais un deuxième choc exogène a rapidement pris le relais, celui des prix pétroliers qui a entraîné tout le secteur de l'énergie à la hausse. Selon l'Office fédéral des statistiques, entre janvier et juillet 2005, la facture des ménages pour l'électricité s'est accrue de $4 \%$, celle du gaz de $6 \%$, et celle de l'essence de $16 \%$. Le fuel domestique s'est même renchéri de $31 \%$. Si les prix de l'énergie restent à leur niveau moyen de la première moitié de l'année, un ménage représentatif devra dépenser en 2005 environ $370 €$ de plus pour ses besoins énergétiques qu'en 2004. Depuis 2000, sa facture énergétique est passée de 3870 à $4687 €$; plus de la moitié en est imputable à l'essence (2 $604 €$, soit $+21 \%$ depuis 2000$), 1428 €$ au fuel $(+16 \%)$ et $655 €$ à l'électricité $(+34 \%)$. La hausse ne concerne donc pas seulement les produits pétroliers.

L'augmentation des prix énergétiques ampute le budget des ménages et affecte leur moral. Les entreprises doivent affronter, outre cette moindre demande intérieure, des coûts de production plus élevés. Compte tenu de la faiblesse de la consommation, la plupart des entreprises n'ose pas répercuter cette hausse sur

Deux chocs exogènes pèsent sur entreprises et ménages...

... mais la croissance n'en pâtit que faiblement 
les clients. Jusque là, elles ont pu financer ces coûts par une hausse soutenue des bénéfices. Si la croissance mondiale reste robuste, cette situation pourra perdurer encore un peu. Pour l'instant, l'impact sur la croissance reste limité. En 2005, il sera très certainement inférieur à 1 point de pourcentage ; la plupart des instituts de conjoncture l'estiment dans une fourchette comprise entre 0,3 et 0,5 point de pourcentage.

Coûts de l'énergie et ménages depuis 2000

\begin{tabular}{|c|c|c|c|c|c|c|}
\hline & 2000 & 2001 & 2002 & 2003 & 2004 & $2005^{1)}$ \\
\hline Dépenses énergétiques par ménage ${ }^{2)}$ & 3870 & nd & nd & nd & 4314 & $4687^{3)}$ \\
\hline Facture totale ${ }^{4)}$ & 35 & 32 & 27 & 29 & 34,4 & nd \\
\hline Consommation privée ${ }^{5)}$ & 2,2 & 1,8 & $-0,7$ & 0,0 & $-0,8$ & 0,4 \\
\hline Rémunération par employé ${ }^{6)}$ & 2,2 & 1,7 & 1,4 & 1,7 & 0,1 & 0,1 \\
\hline Taux d'épargne des ménages ${ }^{7)}$ & 9,7 & 10,2 & 10,5 & 10,7 & 10,9 & 11,1 \\
\hline
\end{tabular}

Source : www.destatis.de, OCDE (2005). (1) Prévision. (2) Dépenses en € pour un ménage moyen. (3) Sur la base des prix et dépenses moyennes du premier semestre 2005. (4) En milliards $€$. (5) Pourcentage de variation par rapport à l'année précédente en volume. (6) dans le secteur des entreprises, pourcentage de variation par rapport à l'année précédente

\section{Ménages et industrie résistent au choc pétrolier}

L'épargne des ménages en hausse

Pas de spirale salaires-prix malgré le pétrole

L'efficacité énergétique accrue freine l'impact de la hausse

La chimie s'en sort bien...
Le risque d'un ralentissement plus marqué n'est néanmoins pas écarté. Les ménages allemands sont déstabilisés face au contexte de restructuration économique général ; toute discussion d'un renchérissement supplémentaire du coût de l'énergie risque de les ébranler davantage. Par ailleurs, la confiance des ménages allemands est particulièrement sensible aux pertes futures possibles d'emploi et de revenu. Cette crainte entraîne à la hausse l'épargne de précaution (avec 11,1\%, elle est néanmoins toujours en dessous des $13 \%$ du début des années 1990) et comprime ainsi davantage la demande intérieure.

Mais malgré cette frilosité de la demande, nous sommes très loin des chocs des années 1970/80. Une des raisons est le temps d'ajustement des prix. Par exemple, lors de la crise de 1973/74, les prix ont triplé sur une période de trois mois. Cette fois-ci, il leur a fallu trois ans et demi pour ce même parcours, laissant ainsi aux entreprises et ménages du temps pour s'adapter. Ce temps d'ajustement plus long, mais également un comportement modifié des syndicats qui commencent, à l'instar d'IG Metall, à considérer la question des salaires selon une approche de compétitivité globale (voir REA 72/05), expliquent pourquoi on n'observe pas en Allemagne de spirale salaires-prix. Au milieu des années 1970, les salaires avaient grimpé de plus de $10 \%$, et les prix entre 6 et $7 \%$. Aujourd'hui, la situation est tout autre : en juillet 2005 , les salaires fixés dans le cadre des conventions collectives ont enregistré une augmentation de seulement $1 \%$ sur douze mois - moins que l'évolution générale des prix (+2\%). L'augmentation a été la plus forte pour les salariés dans le secteur de la machine-outil et de la construction navale (+2\%), ainsi que dans la métallurgie $(+1,9 \%)$; elle a été de seulement $+0,4 \%$ dans le commerce.

Plus fondamentaux sont les changements dans les modes de production. L'industrie allemande produit aujourd'hui avec nettement moins d'énergie par unité de production que dans les années 1970. Elle produit aussi des machines plus économes dont la demande s'accentue naturellement en période de hausse des coûts énergétiques. Depuis 1980, l'efficacité énergétique de l'industrie s'est nettement améliorée. Certes, en 2004, la facture allemande (entreprises et ménages) pour l'achat de pétrole (34,4 milliards $€$ ), avait presque rejoint son niveau le plus haut : 36,2 milliards $€$ en 1981. Mais si on la rapporte au PIB, la situation est différente : en 1981, les dépenses pétrolières représentaient $4,6 \%$ du $\mathrm{PIB}$; en 2004, leur part n'est que de 1,6 \% (c'est néanmoins une augmentation de 0,8 point de pourcentage par rapport au niveau le plus bas en 1998).

Malgré cette plus grande efficacité énergétique, le bilan par secteur est contrasté. Pour la chimie, les conséquences négatives restent finalement très limi- 
tées, bien qu'avec 20 millions de tonnes par an (près de $20 \%$ de la consommation allemande), il s'agisse du secteur le plus gourmand en produits pétroliers. Au printemps, la chimie prévoyait une croissance de 2 à $2,5 \%$ en 2005, une estimation basée sur un prix par baril compris alors entre 35 et $40 \$$. Six mois plus tard - et malgré un prix du baril largement au-dessus de ce niveau -, le secteur s'attend même à une croissance de $4,8 \%$, ce qui représente une nette accélération par rapport à $2004(+2,5 \%)$. La chimie se place ainsi bonne deuxième dans le classement sectoriel après l'électrotechnique (+ $8 \%$ prévus).

Les poids lourds du secteur ne sont ainsi guère inquiets. BASF (au bénéfice en hausse de $+29 \%$ sur un an au troisième trimestre 2005) profite même directement de la flambée des prix énergétiques, car l'entreprise n'est pas seulement consommatrice de pétrole, elle offre également des produits pétroliers et gaziers à travers sa filiale Wintershall. Pour Bayer aussi, les répercussions sont limitées, car l'entreprise offre beaucoup de produits très riches en $R \& D$, ce qui fidélise de fait les clients (ils ne peuvent guère changer rapidement de fournisseur) et permet ainsi à l'entreprise de répercuter l'augmentation des coûts de production plus facilement aux clients.

La situation du secteur automobile est très différente. Comme le tourisme et l'aéronautique, il est directement touché par l'augmentation des prix des carburants. Après une expansion de $4 \%$ en 2004, le secteur devra se contenter en 2005 d'une croissance plus faible $(+1,5 \%)$. Un des handicaps est que les producteurs allemands n'ont ni un avantage comparatif dans le segment des véhicules à faible consommation d'essence (depuis 1990, pour l'ensemble des fabricants allemands, la consommation moyenne d'essence a baissé de $21,2 \%$; celle de Renault, de $12,5 \%$ sur 10 ans) ni dans les modèles à énergie alternative. Dans le domaine des voitures hybrides (moteur électrique et à combustion traditionnelle), le retard pris sur les concurrents japonais est considérable. Tandis que Toyota vend son modèle hybride outre-Rhin depuis 2004, les constructeurs allemands en sont encore à la phase de conception. De plus, ils visent d'abord le marché américain afin d'y maintenir leurs parts de marché. Enfin, les voitures à hydrogène, annoncées par les constructeurs allemands pour la fin des années 1990, ne seront produites en série que vers... 2010.
... notamment grâce à son offre de produits pétroliers

L'automobile souffre, ....

Situation sectorielle dans l'industrie (1)

\begin{tabular}{|l|cc|cc|cc|cc|}
\hline & \multicolumn{2}{|c|}{ Production } & \multicolumn{2}{|c|}{ Coûts de production } & \multicolumn{2}{c|}{ Emploi } & \multicolumn{2}{c|}{ Taux exportateur } \\
& $\mathbf{2 0 0 4}$ & $\mathbf{2 0 0 5}$ & $\mathbf{2 0 0 4}$ & $\mathbf{2 0 0 5}$ & $\mathbf{2 0 0 4}$ & $\mathbf{2 0 0 5}$ & $\mathbf{2 0 0 4}$ & $\mathbf{2 0 0 5}$ \\
Industrie chimique & 2,5 & $\mathbf{4 , 8}$ & 1,0 & 3,5 & $-4,1$ & $-0,8$ & 66,7 & 66,8 \\
Métallurgie & 3,0 & 0,8 & 6,9 & 6,5 & $-0,7$ & 0,0 & 38,8 & 39,5 \\
Machine-outil & 3,5 & 3,8 & 1,0 & 1,5 & $-1,5$ & $-1,3$ & 61,0 & 63,0 \\
Construction automobile & 4,0 & $\mathbf{1 , 5}$ & 0,8 & $\mathbf{1 , 5}$ & 0,5 & 0,3 & 48,4 & 50,0 \\
Total de l'industrie & $\mathbf{3 , 2}$ & $\mathbf{3 , 0}$ & $\mathbf{1 , 7}$ & $\mathbf{2 , 3}$ & $\mathbf{- 1 , 9}$ & $\mathbf{- 1 , 0}$ & $\mathbf{4 7 , 2}$ & $\mathbf{4 8 , 5}$ \\
\hline
\end{tabular}

Sources : www.destatis.de; Commerzbank Research (2005). (1) Variation par rapport à l'année précédente. Estimation pour 2005.

A l'inverse, dans la construction mécanique, traditionnel fleuron de l'économie allemande, les ventes sont très soutenues. L'augmentation de la production du secteur se stabilisera en 2005 à un niveau élevé $(+3,8 \%$, après $3,5 \%$ en 2004). Conséquence directe du renchérissement des coûts énergétiques, les machines pour l'énergie mécanique, l'exploitation minière, ainsi que pour l'agriculture et la sylviculture figurent en tête des ventes en 2005. Le taux exportateur de la branche (pourcentage du volume des exportations par rapport à l'ensemble du chiffre d'affaire) est estimé à $63 \%$ pour 2005 (après $61 \%$ en 2004). Les entreprises du secteur vont ainsi consolider leur place de champion mondial en termes de part de marché (19,3\% du marché mondial en 2004). Comme pour l'ensemble de l'industrie allemande, le principal facteur de soutien est la demande extérieure, notamment celle de la Chine et des pays producteurs de pétrole. Mais malgré ces bonnes performances, la construction mécanique
... mais pas la construction mécanique 
La demande chinoise change la donne

n'apporte pas, elle non plus, de contribution positive au marché de l'emploi ; l'emploi industriel continue de reculer.

\section{Exportations : Chine, pétrodollars et taux de change réel}

Les craintes d'une récession due à la hausse du pétrole se nourrissent des expériences du milieu et de la fin des années 1970. Or contrairement à cette époque où la hausse des prix énergétiques était consécutive à un resserrement de l'offre, les prix sont aujourd'hui poussés par la demande, notamment celle de la Chine. Car le pétrole est le véritable lubrifiant pour la formidable croissance chinoise et permet à son économie de s'intégrer dans la division internationale du travail. L'Allemagne est un grand bénéficiaire de cet essor. D'abord, les entreprises allemandes profitent d'une demande chinoise de plus en plus forte. Certes, les Chinois ont acheté en 2004 à peine un tiers de ce que les Américains achètent outre-Rhin ; mais avec $+15 \%$, les exportations vers la Chine ont progressé si fortement que leur contribution à la croissance totale des exportations allemandes en 2004 était équivalente à celle des ventes aux Etats-Unis. Compte tenu de cette dynamique exportatrice, la Chine est aujourd'hui un débouché aussi important pour l'Allemagne que les US (voir REA 70/05).

Les prix continuent de n'augmenter que faiblement

\begin{tabular}{|c|c|c|c|c|c|}
\hline Variations en \% & $2002^{1)}$ & $2003^{1)}$ & $2004^{1)}$ & Variation des composantes des coûts production $2005^{2)}$ & \\
\hline Coûts de production & $-0,6$ & 1,7 & 1,6 & Energie $(19,7 \%):+16,5 \%$ & $\begin{array}{l}\text { des couts } \\
\text { production }\end{array}$ \\
\hline Prix d'importation & $-2,2$ & $-2,2$ & 1,0 & Biens intermédiaires $(30,6 \%):+2 \%$ & $2005^{2)}$ \\
\hline Prix à la consommation & 1,4 & 1,1 & 1,6 & $\begin{array}{l}\text { Biens de consommation }(25,3 \%):+1,2 \% \\
\text { Biens d'investissement }(24,4 \%):+0,7 \%\end{array}$ & $+4,6 \%$ \\
\hline
\end{tabular}

Source : www.destatis.de; IW (2005). (1) Variation par rapport à l'année précédente. (2) Entre parenthèses, la pondération des différentes composantes de l'indice des prix producteurs. Données pour des biens produits et vendus en Allemagne en août 2005 par rapport à août 2004. (3) Données pour des biens produits et vendus en Allemagne en août 2005 par rapport à août 2004.

Exportations en hausse et prix à l'importation en baisse

Le recyclage des pétrodollars soutient l'économie mondiale

L’Allemagne : premier bénéficière du recyclage des pétrodollars
L'Allemagne profite par ailleurs d'importations chinoises à des prix très bas. Le même effet est exercé par les pays de l'Europe de l'Est. La conséquence est que les prix pour des biens négociables au niveau international n'augmentent que très faiblement. Pour 2005, avec une facture énergétique de plus en plus élevée, on s'attend néanmoins à une accélération (entre 3 et $4 \%$, après $1,6 \%$ en 2004 pour les coûts de production). Des exportations en hausse et des biens d'importations moins chers - ces deux tendances sont directement liées à l'augmentation du prix du pétrole et aident à limiter ses conséquences négatives. Une économie avec un degré d'ouverture comme celui de l'Allemagne s'accommode probablement mieux d'une croissance chinoise forte et de prix pétroliers élevés que d'une Chine à la croissance faible et d'un pétrole à prix modéré (voir REA 63/03).

Outre la dynamique chinoise, c'est le recyclage de la rente pétrolière qui stimule fortement les exportations allemandes. Contrairement au début de la phase de hausse où les pays producteurs de pétrole (OPEP et CEI qui assurent $70 \%$ des exportations mondiales de pétrole) avaient affecté une large part de leurs surplus aux dépôts bancaires, depuis 2001, ils les re-investissent dans le circuit économique mondial. Une partie va vers les marchés financiers et contribue ainsi à contenir les taux d'intérêt, l'autre partie est utilisée pour l'achat de biens d'investissement et de consommation. La zone euro bénéficie plus que d'autres régions de cette tendance : entre 2001 et 2004, ses exportations vers l'OPEP et la CEI ont progressé de $22 \%$ et $34 \%$ respectivement, améliorant ainsi la part de marché de la zone euro de 24,8 à $27,1 \%$ et de 31,6 à $39,6 \%$ respectivement (dans le même temps, celle des Etats-Unis baissait dans les deux zones).

En Europe, l'Allemagne profite particulièrement de cette tendance. Selon une estimation du ministère fédéral de l'Economie, les exportations allemandes vers les pays de l'OPEP croissent de $13 \%$ quand ces pays bénéficient d'une hausse 
des recettes pétrolières de $10 \%$. L'Allemagne profite davantage du recyclage des pétrodollars que ses concurrents parce qu'elle offre la panoplie de produits recherchée par ces pays, surtout des produits d'investissement. Ainsi, les exportations allemandes vers les pays du Proche et Moyen Orient ont progressé de $37 \%$ entre 2001 et 2004, celles vers la Russie de 38,9\%, dépassant pour les deux régions la moyenne des exportations allemandes (+20,8\% entre 2001 et 2004). Cette évolution dynamique se poursuit en 2005.

Les exportations allemandes profitent de la hausse du pétrole ${ }^{1)}$

\begin{tabular}{|lrrrc|}
\hline Variations en \% & $\mathbf{2 0 0 2}$ & $\mathbf{2 0 0 3}$ & $\mathbf{2 0 0 4}$ & $\mathbf{2 0 0 5}^{\mathbf{2}}$ \\
Vers le Proche et le Moyen Orient & 7,0 & 3,1 & 24,3 & 16,4 \\
Vers la Russie & $-4,4$ & 6,6 & 36,3 & 10,9 \\
Vers la Chine & 20,2 & 25,3 & 15,1 & $n d$ \\
Total des exportations & 7,7 & 2,0 & 10,0 & 5,5 \\
Prix d'un baril en dollars $^{3)}$ & $-7,1$ & 13,1 & 33,8 & 35,5 \\
\hline
\end{tabular}

Source : www.destatis.de, IW (2005). (1) Variations par rapport à l'année précédente. (2) Variation des exportations de biens entre janvier et juillet 2005 par rapport à la même période 2004. (3) Variation du prix moyen sur l'année par rapport à l'année précédente ; pour 2005 : sept premiers mois.

En plus de cette double stimulation, les entreprises allemandes profitent du cadre institutionnel de l'UEM. Avec un niveau des taux d'intérêt à court terme fixé à l'identique pour tous les 12 membres, mais des taux d'inflation différents, on aboutit à des taux d'intérêt réels qui varient d'un pays à un autre. L'Allemagne, avec son taux d'inflation comparativement faible, obtient ainsi des taux d'intérêt réels relativement élevés. On trouve ici un des facteurs qui expliquent la faiblesse persistante de la demande intérieure. Mais le niveau d'inflation relativement faible en Allemagne représente également un atout considérable, car il se traduit par une dévaluation réelle par rapport aux autres pays européens : les prix des produits allemands augmentent moins vite que ceux des concurrents européens. Ce taux de change réel plus faible stimule les exportations ; il contribue ainsi à expliquer l'excellente performance des entreprises allemandes à l'étranger. L'UEM produit ainsi un double effet et à signe opposé pour l'Allemagne. L'effet cumulé devrait être plutôt bénéfique pour l'économie allemande compte tenu du fait que la croissance allemande dépend traditionnellement moins de la consommation qu'en France ou aux USA.

Les données fournies par le Conseil des Sages sont sans appel : pour la période 2001-2004, les exportations allemandes ont augmenté deux fois plus vite que celles de la zone euro (et six fois plus vite que celles de la France); en même temps, l'effet cumulé par rapport au taux d'intérêt réel s'établit à 1,75\% pour l'Allemagne $(0,75 \%$ pour la zone euro et $1,2 \%$ pour la France). Du côté du taux de change réel, la dévaluation du taux de change réel atteint $-5,5 \%$ pour l'Allemagne. Dans la zone euro, seule la Finlande $(-0,9 \%)$ profite également d'une amélioration de sa position extérieure (celle de la France se détériore de 0,5\%, et celle de l'Italie même de 7,2\%).

Cette tendance est corroborée par l'évolution des coûts salariaux unitaires. Depuis 1999, ces coûts ont reculé de plus de $10 \%$ en Allemagne, face à une stabilité en France et une hausse de près de $10 \%$ en Italie et en Espagne. L'affaiblissement des syndicats, combiné au renforcement de la position des entreprises suite aux pressions exercées par la mondialisation et la menace de délocalisations, a ouvert une fenêtre d'opportunité aux entreprises allemandes qui leur a permis de consolider la compétitivité du Standort Deutschland: elle se trouve aujourd'hui à son plus haut niveau depuis plus de dix ans.

\section{La menace des prix élevés de l'électricité et du gaz}

Outre l'appréciation de l'euro et la flambée des prix du pétrole, entreprises et ménages doivent supporter un troisième facteur de coûts : le niveau très élevé
L'UEM aide

l'économie allemande

Exportations en hausse grâce au taux de change réel plus bas...

... et des coûts salariaux en baisse

D'abord baisse, puis hausse du prix de l'électricité 
Engagements internationaux et imposition poussent les prix

des prix pour l'électricité et le gaz. Contrairement aux deux premiers, ce dernier facteur relève pour une bonne partie de raisons internes. Du côté de l'électricité, les prix avaient d'abord entamé une baisse significative après la libéralisation du marché en 1998 (baisse de 40 et $20 \%$ respectivement entre 1998 et 2000 pour les entreprises et les ménages). Mais depuis 2001, les prix augmentent à nouveau, conduisant à une situation franchement défavorable pour l'Allemagne : actuellement, côté ménages (3 $500 \mathrm{kWh}$ par an), seuls les Danois et les Italiens doivent payer plus ; côté entreprises, seule l'Italie a des prix encore plus élevés.

Plusieurs raisons expliquent ce niveau. D'un côté, ce sont des facteurs communs à tous les pays, notamment la hausse du prix des matières premières, les intempéries climatiques (comme les sécheresses au sud de l'Europe) ou le marché des certificats d'émissions, autorisé depuis janvier 2005. Ainsi, sur les marchés d'électricité, la tendance est partout à la hausse : à la bourse EEX de Leipzig, les prix ont grimpé de plus de $25 \%$ depuis le début 2005 ; le même phénomène s'observe sur les autres places européennes. Mais ces facteurs ne suffisent pas à expliquer le niveau plus élevé pour les utilisateurs finaux en Allemagne. Il est gonflé par un fort niveau d'imposition.

Prix de l'électricité et du gaz en Europe $(c t / k W h)^{1)}$

\begin{tabular}{|ll|ll|ll|ll|}
\hline \multicolumn{3}{c|}{ Electricité ${ }^{2)}$} & \multicolumn{3}{c|}{ Gaz $^{3)}$} \\
Italie & 9,18 & France & & & \\
Allemagne & 8,40 & Pologne & 4,90 & Danemark & 2,10 & Pologne & 1,63 \\
Irlande & 7,61 & Suède & 3,82 & Allemagne & 2,04 & Grande Bretagne & 1,58 \\
\end{tabular}

Source : Eurostat. (1) Pour les entreprises, au $1^{\mathrm{er}}$ janvier 2005. (2) Prix avec impôts spécifiques inclus, mais sans TVA ; données au $1^{\text {er }}$ janvier 2005 sur la base d'une consommation de $50 \mathrm{GWh} / 5000 \mathrm{~h} / \mathrm{a}$. (3) 250 jours, 4000 h/116,3 GWh. (4) Pour une consommation annuelle de $24 \mathrm{GWh}$.

Soutien aux énergies renouvelables, un avantage comparatif

L'effet douteux de la loi EEG cumulée aux certificats d'émission
Entre 1998 et 2004, le niveau de la charge fiscale dans le prix payé par les ménages est passé de $25 \%$ à près de $40 \%$; pour les entreprises, l'augmentation a été plus faible (de 2 à $9 \%$ ). Une série de lois et des taxes spécifiques sont responsables de cette hausse: Erneuerbare-Energien-Gesetz (EEG), KraftWärmekopplungs-Gesetz (KWG), Konzessionsabgabe et impôt d'électricité. Avant TVA, les utilisateurs versent 12 milliards $€$ à l'Etat. Impôts et autres prélèvements représentent ainsi le premier poste dans le prix payé par les ménages $(39,3 \%)$. Le reste se partage entre frais de réseau (Netzentgelte ; 32,8\%), le coût de la production (16,4\%), ainsi que celui de la distribution et de la mesure $(11,5 \%)$. Mais même si cet arsenal peut paraître énorme, la part propre au soutien des énergies renouvelables est très faible : la part de la loi EEG ne représente que 3,8\% du prix (données pour un ménage moyen en 2003).

Cette hausse de la charge fiscale a été le fruit de décisions politiques (voir REA 72/05) afin de permettre à l'Allemagne de respecter le protocole de Kyoto. Ensuite, elles représentent un soutien direct au développement des énergies renouvelables. L'Allemagne détient aujourd'hui une place de leader dans ce domaine, et les solutions industrielles figurent parmi les meilleures ventes, notamment pour les entreprises du Mittelstand (voir REA 57/02). Consommateurs et entreprises subventionnent ainsi des emplois en Allemagne dans le secteur de la photovoltaïque, des éoliennes ou de la biomasse. Ce phénomène a également atteint la bourse de Francfort, où les entreprises solaires enregistrent des progressions record. Actuellement, rien n'indique que le soutien aux énergies renouvelables disparaîtra rapidement. Le nouveau gouvernement a annoncé son intention de faire progresser la part des énergies renouvelables dans la production d'électricité d'environ $10 \%$ aujourd'hui à au moins $12,5 \%$ en 2010 et à $20 \%$ en 2020. Le loi EEG restera ainsi en vigueur mais son efficacité sera évaluée avant 2007.

Si aujourd'hui la loi EEG s'est transformée en soutien pour le Mittelstand, sa raison d'être première (et politique) est la réduction des émissions de $\mathrm{CO}_{2}$. Ce but 
n'est cependant pas atteint : l'existence conjointe de la loi EEG et du marché des certificats d'émission a comme effet que le volume global de $\mathrm{CO}_{2}$ ne baisse pas. En effet, si un producteur d'électricité décide de passer à la production par énergies renouvelables, il vend ses certificats sur le marché des permis. Suite à l'accroissement de l'offre, le prix des certificats baisse. Certes, dans le secteur producteur d'électricité, l'émission de $\mathrm{CO}_{2}$ s'est réduite, mais la baisse du prix des certificats permet à d'autres branches industrielles d'en acheter plus et de réduire leurs investissements dans des techniques plus propres. Puisque les certificats sont négociés sur un marché européen, l'ensemble des entreprises européennes profite ainsi de la loi EEG allemande. Cette loi subventionne ainsi tout le secteur émetteur de $\mathrm{CO}_{2}$, mais sans réduire le volume total de $\mathrm{CO}_{2}$ car le nombre de certificats reste au même niveau. Pour rendre vraiment efficaces des instruments tels la loi EEG, il faudrait que leur introduction s'accompagne d'une réduction des certificats - autrement dit de l'engagement inscrit dans le protocole de Kyoto.

Aussi fondamental pour le niveau élevé des prix que ces choix politiques est le fait que la libéralisation de 1998 n'a pas été complète. Elle a surtout épargné les big players (E.on, EnBW, RWE) du marché allemand. Conséquence: au moment où tous les acteurs se plaignent du niveau élevé des prix de l'énergie, les entreprises productrices d'électricité réalisent des bénéfices records. Entre janvier et juin 2005, le bénéfice des trois premières a ainsi dépassé de plus de $100 \%$ la somme totale dépensée en 2004 pour le soutien aux énergies renouvelables. Malgré la libéralisation formelle, les grandes entreprises productrices d'électricité profitent toujours d'un quasi-monopole : leur position de leader leur permet d'exiger des frais de réseaux parmi les plus élevés en Europe - et ceci malgré la modernité des réseaux et l'absence d'une topographie particulièrement défavorable.

Ce même constat d'un niveau de concurrence encore insuffisant s'applique au marché allemand du gaz, où les prix sont artificiellement tirés vers le haut par l'indexation du prix du gaz sur celui du pétrole, un système qui a certainement fait son temps. Néanmoins, la concurrence fait lentement son chemin. L'Office fédéral des cartels a ouvert une enquête contre les producteurs de gaz soupçonnés de pratiquer des prix artificiellement élevés. Même situation du côté d'E.on et de RWE. Chez eux, l'Office s'intéresse au rôle joué par les certificats d'émission dans le calcul des prix d'électricité. On reproche aux producteurs d'intégrer le prix de ces certificats dans les prix finaux - et cela malgré le fait que les entreprises avaient initialement reçu gratuitement ses droits à polluer.

Le problème n'est cependant pas uniquement du côté allemand. Malgré la libéralisation formellement très avancée - dans cinq pays européens, dont l'Allemagne et le Royaume Uni, les marchés de l'électricité et du gaz sont à $100 \%$ ouverts à la concurrence ; dans six autres pays, le taux d'ouverture varie entre 56 et $100 \%$ - nous sommes encore loin d'un marché européen de l'énergie. Le commerce intraeuropéen d'électricité est encore très faible, notamment à cause d'une interconnexion insuffisante au niveau des frontières. Ainsi, en Allemagne, la part du commerce d'électricité n'est passée que de 12 à $16 \%$ entre 1992 et 2002 (par rapport à la production totale). En France, l'évolution est encore moins bonne. Visiblement, les producteurs des deux plus grands Etats de l'UE n'ont pas intérêt à investir dans le commerce intracommunautaire : ils craignent un nivellement des prix vers le bas compte tenu des grandes différences des prix en Europe.

Depuis le $1^{\text {er }}$ juillet 2005, la nouvelle loi de gestion de l'énergie (Energiewirtschaftsgesetz, EWG), qui transpose les orientations d'une directive européenne de 2003 visant à accélérer la libéralisation des marchés énergétiques européens, cherche à améliorer la situation pour les consommateurs, notamment dans le domaine des frais de réseaux et du choix du fournisseur. L'application de cette loi sera une des tâches principales de la nouvelle Bundesnetzagentur
Le marché pénalisé par un manque de concurrence

Gaziers et électriciens sous l'œil de l'Office fédéral des cartels

Un marché européen de l'énergie encore sous-développé

Les premiers signes d'une amélioration 
(Office fédéral de réseaux), l'ancienne Agence de régulation de la poste et des télécommunications qui a vu ses compétences étendues au secteur énergétique. Une autre sera la déconcentration d'entreprises qui réunissent production et distribution d'électricité, comme le souhaite la Commission européenne. La situation peut ainsi évoluer favorablement pour le consommateur. En octobre dernier, la Cour suprême de cassation allemande (Bundesgerichtshof) a décidé pour sa part que les offreurs d'électricité peuvent exiger que les propriétaires des réseaux communiquent le calcul des frais de réseau - facteur prépondérant dans le coût d'électricité d'outre-Rhin.

SI ON CONSIDERE LES RESULTATS des entreprises, notamment à l'export, l'économie allemande va mieux - et ceci malgré la conjonction de plusieurs facteurs défavorables, notamment dans le domaine de l'énergie. Les derniers indices ifo confirment cette orientation : le climat des affaires s'est nettement amélioré en octobre 2005, il atteint son niveau le plus haut depuis 2001 ; même le moral des ménages commence à s'embellir. Mais pour que cette prospérité des entreprises engendre également la prospérité du site Allemagne en général, le nouveau gouvernement est appelé à y contribuer. A côté des questions prioritaires telle que la consolidation des finances publiques, il doit également veiller aux conséquences du formidable succès des entreprises allemandes à l'étranger qui témoigne certes de la vitalité des entreprises, mais pas forcément de celle de l'économie allemande.

Cette hypothèse, qui fait débat outre-Rhin sous la notion un peu malheureuse de Basarökonomie (économie de bazar), reflète néanmoins une certaine réalité. Suite à la présence croissante de biens intermédiaires étrangers, la création de valeur réalisée sur le sol allemand diminue : entre 1991 et 2002, la contribution étrangère (biens intermédiaires et importations destinées directement à l'exportation) à la création de valeur allemande est passée de $26,7 \%$ à $38,8 \%$. II est encore trop tôt pour interpréter définitivement cette tendance : elle peut aussi bien exprimer un appauvrissement de la base industrielle allemande qu'une insertion plus efficiente des entreprises allemandes dans la division internationale du travail. Cependant, cette grande imbrication commerciale pousse le commerce extérieur à elle seule : une voiture fabriquée à $88 \%$ à Bratislava et finalisée à Leipzig, comme c'est le cas pour la Porsche Cayenne, entre à $100 \%$ dans les statistiques allemandes du commerce extérieur. Cela explique pourquoi l'effet marginal des exportations sur le produit national allemand n'est que de $45 \%$ : un euro à l'exportation n'augmente le PIB que de $0,45 €$. Mais quoi qu'il en soit, la contribution du commerce extérieur à la croissance allemande reste vitale : l'excédent commercial a apporté près de $6 \%$ au PIB en 2004.

\section{Indications bibliographiques :}

AUTRET F., «Energie : une politique énergétique entre compétitivité et environnement », Regards sur l'économie allemande, $n^{\circ} 72,2005$

COMMERZBANK RESEARCH, "Deutschland : Branchenreport Industrieprognose », septembre 2005

DiekMANN J. et al., « Energiepreise in Bewegung », DIW Wochenbericht 44/2004

GABEL M., "Commerce extérieur: Chine, Inde, Brésil - les choix des entreprises allemandes ", Regards sur l'économie allemande, $\mathrm{n}^{\circ} 70$, mars 2005 ; "Le commerce extérieur : atouts et faiblesses ", Regards sur l'économie allemande, n63, octobre 2003 et « Développement durable et éco-industries ", Regards sur l'économie allemande, $n^{\circ} 57$, juillet 2002 OCDE (2005), Perspectives économiques de l'OCDE, vol. 2005/1, n7, juin 2005

SACHVERSTÄNDIGENRAT ZUR BEGUTACHTUNG DER GESAMTWIRTSCHAFTLICHEN ENTWICKLUNG, Jahresgutachten 2004/5 (12-11-04) et Jahresgutachten 2005/6 (9-11-05)

"Preise und Kosten : Energie als Treiber », iwd 41/05

RICHMANN A., «Energiepreise auf Spitzenniveau - Standortnachteile für die deutsche Industrie », Ifo Schnelldienst, 19/05 